\title{
Expert recommendations on the management of childhood bronchial asthma during the novel coronavirus pneumonia epidemic
}

\author{
China Association for Promotion of Health Science and Technology Child Allergy Professional \\ Committee
}

Correspondence to: Qinglong Gu. Children’s Hospital Affiliated to Capital Institute of Pediatrics, Beijing, China. Email: gq171@163.com; Chuangli Hao. Children's Hospital of Soochow University, Suzhou, China. Email: hcl_md@163.com.

Submitted Apr 16, 2020. Accepted for publication Jul 28, 2020.

doi: $10.21037 /$ jtd-20-1688

View this article at: http://dx.doi.org/10.21037/jtd-20-1688

Novel coronavirus pneumonia (coronavirus disease 2019, COVID-19), first identified in December 2019, is caused by severe acute respiratory syndrome coronavirus 2 (SARS$\mathrm{CoV}-2)$ (1). SARS-CoV-2 is sensitive to ultraviolet light and temperature, and can be inactivated by disinfectants, e.g., exposure to $75 \%$ ethanol for 30 minutes at $56^{\circ} \mathrm{C}$. SARS$\mathrm{CoV}-2$ is highly infectious and all populations are equally susceptible, including children (2). As of 12 April 2020, a total of 82,160 cases of COVID-19 had been confirmed in China, and 3341 patients had died (3). Childhood asthma is the most common chronic airway disease in children. During the COVID-19 outbreak, children with asthma may be more susceptible to SARS-CoV-2 infection due to autoimmunity, long-term use of certain drugs and coexisting diseases. Childhood asthma is a chronic respiratory disease that can be exacerbated by environmental pollutants, infectious organisms and psychological factors. Equally, as most children were required to stay at home during the epidemic, exposure to indoor triggers, including dust, anxiety, decreased exercise and reduced visits to the clinic/ follow-up, makes the effective management of asthma more difficult. In response to the COVID-19 outbreak, the Paediatric Committee for Allergy, China Association for Promotion of Health Science and Technology convened a panel of experts to put forward suggestions for prevention, environmental intervention, family management, psychological intervention and reasonable exercise for children with asthma, which can be used as a reference for pediatric clinicians and parents.

\section{Prevention of SARS-CoV-2 infection in children with asthma}

\section{Risk of infection in children with asthma}

Immune imbalance in children with asthma

Imbalance of T helper (Th) 1 and Th2 cells as well as changes in cytokine levels such as interleukin (IL)-10, IL6, IL-4, IL-17 and interferon (IFN)- $\gamma$ ) are observed in patients with asthma. These changes increase the risk of infection by bacteria, viruses, fungi, and other pathogenic microorganisms, especially in children (4).

\section{Drug-related risk of infection}

Inhaled corticosteroids (ICS) are the first choice for the treatment of asthma, with a low incidence of adverse reactions and a good safety profile. Long-term high-dose use of ICS can reduce levels of IL-2, T cell proliferation, phagocytosis and lymphocyte function. Importantly, oral fungal infection may occur in some children due to the improper use of ICS. These systemic and local side effects increase the risk of infection, and repeated use of systemic glucocorticoids in some children with severe asthma increases the risk of infection (5).

\section{Prevention}

Severe acute respiratory syndrome coronavirus 2 (SARS-CoV-2) is easily spread among individuals, and asymptomatic patients can also spread the virus. 
Respiratory droplets and close contact are the main routes of transmission; however, the opportunity for aerosol transmission increases with close proximity over time due to high virus concentration.

Most cases of SARS-CoV-2 infection in children are caused by close family contact; therefore, parents and relatives should ensure adequate protection by not going out as much as possible, avoiding crowds, avoiding close contact with anyone who has respiratory symptoms, and avoiding public transport. Relatives should wear a mask when going out or taking public transport, and should change shoes, wash hands (seven-step hand-washing method) and change clothes after returning home and before touching children. They should also pay attention to cough etiquette, covering the nose and mouth with a paper towel when sneezing or coughing. In addition, keeping the room and workplace clean, opening windows frequently, and keeping rooms well ventilated also reduce the risk of virus transmission. Parents should drink more water, rest well and consume a reasonable diet. In addition, breastfeeding mothers should wear a mask, wash their hands, and maintain good personal hygiene.

Children should avoid going out as far as possible, wash their hands frequently and avoid touching their faces, especially the eyes or nose. In addition, children should not lick their fingers or touch anything unnecessarily. Moderate exercise, a reasonable diet, and adequate sleep are very important. It is also recommended that children wear special masks that meet national standards, and it is not recommended that children wear adult masks that do not fit properly.

When beginning a new semester, group exposure risk increases; therefore, more attention should be paid to protect against viral infection. Children should walk to school if possible, and wear a mask if they had to take public transport. Children should wash hands frequently and avoid touching anything in public. Paper towels should be used to mask the hand from the object if contact is necessary. Daily checks should be performed on students' physical condition, and suspicious symptoms should be reported on time. Large group activities should be avoided and ventilation should be maximized in classrooms, dormitories, libraries, toilets and other public areas $(2,6)$.

\section{Indoor environmental risks and interventions in children with asthma during the COVID-19 epidemic}

Environmental factors play an important role in the development of asthma. Among them, the indoor environment has been known to induce acute asthma attacks. Whilst During COVID-19 was prevalent pandemic, most children stayed indoors; therefore, the effects of indoor environmental factors on childhood asthma increased significantly.

\section{Indoor allergen exposure and intervention}

Indoor allergens are able to induce acute asthma attacks in children. Dust mites (including household dust mites) are the most common indoor inhalation allergens. Other common indoor allergens associated with acute attacks in childhood asthma include mold, cockroaches, pet dander and excrement (7).

Specific interventions can help to protect against different types of allergen: for example, such as anti-mite bedspreads, hypoallergenic pillowcases and other household appliances can be used to protect against different types of allergens. Bedding, pillows, and sheets, etc. should be washed once every two weeks. Children should avoid jumping on the bed and staying from plush toys. Households with cats and dogs should be as well ventilated as possible. Air purifiers should be used where available and air conditioners/humidifiers should be avoided (8).

\section{Interventions against physical and chemical factors}

Physical and chemical stimuli can lead to acute attacks of asthma induced by airway spasm. Common physical and chemical stimuli include haze, lampblack, second-hand cigarette smoke, disinfectants, perfume, air fresheners and mosquito repellent incense.

The use of disinfectants should be reduced/avoided during the COVID-19 epidemic. Studies have shown that disinfectant can aggravate type 2 cytokines that mediate inflammation and airway hyperresponsiveness (9). Especially for preschool children, short-term exposure to disinfectant particles may cause lung injury and induce acute attacks and deterioration of asthma. Disinfectant inhalation not only induces acute attacks, but also affects the development of asthma. In addition, exposure to disinfectants can aggravate asthma attacks by sensitizing patients to common air allergens. During the COVID-19 epidemic, guidelines widely recommend the use of disinfectants. As such, the significantly increased use of indoor and outdoor disinfectants may have a profound impact on children with asthma by increasing the risk of acute attacks. 
Families of asthmatic children must use disinfectants correctly. They should try not to use chlorine-containing disinfectant, or mix chlorine-containing disinfectant with other disinfectants or cleaners to avoid significant chlorine release. Disinfectant wipes are preferred and disinfection spray should be avoided. Chlorine-containing disinfectants are used once a day at the most. All windows should be open for ventilation during use. Children should not be present when disinfectant is in use and can only be present after adequate ventilation. If children are highly sensitive to disinfectants, ultraviolet irradiation is recommended once a day for no less than 30 minutes.

Keeping indoor airflow is suggested during the COVID-19 epidemic. Ventilation is effective to reduce exposure to indoor allergens, disinfectants, and other physical and chemical factors, also it is an important measure to reduce indoor virus concentration and prevent infection. Generally, the window should be open for ventilation 2-3 times a day, each time for no less than 30 minutes, and different rooms should be ventilated in turn. It is suggested to move sick children to another room when ventilating, and keep children warm to avoid infection during ventilation.

\section{Management of the acute and remission period in children with asthma attack during the COVID-19 epidemic}

\section{Management of acute asthma}

\section{Evaluation of acute attacks and principles of home management}

During the COVID-19 epidemic, individualized treatment should be adopted in children who experience an acute asthma attack based on the original treatment plan according to age, severity of the attack, and concurrent medical conditions. Parents should pay close attention to children's symptoms, and contact their attending doctor as much as possible to discuss the best treatment. Mild attacks can be treated in the family, whereas moderate-to-severe attacks need to be treated in hospital in good time.

If symptoms such as cough and sneeze are observed in children with asthma, ICS such as budesonide suspension ( $1 \mathrm{mg}$ twice a day, 7 days in a row) can be used at home to prevent the occurrence of an acute asthma attack.

When wheezing occurs, the severity should be evaluated. Mild attacks generally do not affect children's activities; if children can speak in full sentences, treatment with an inhaled, short-acting $\beta 2$ receptor agonist should be the first choice, e.g., salbutamol/terbutaline aerosol. If a combination of short-acting bronchodilator and high-dose ICs are available as initial treatment, the total course of treatment should be maintained for at least 7-10 days. For moderate or severe acute attacks with observed dysphoria, anxiety or drowsiness, acceleration of respiration and/or heart rate, dyspnea, inability to speak, cyanosis of lips, or difficulty in lying; or cases with a poor response after initial treatment for a mild attack, for instance, the maintenance time less than $4 \mathrm{~h}$, an inhaled short-acting bronchodilator and high dose ICS should be used together, and patients should seek emergency treatment immediately.

\section{Drug treatment during acute attacks \\ Inbaled short-acting $\beta 2$ receptor agonist}

Inhaled short-acting $\beta 2$-agonists are the first-line drug of choice in treating acute asthma attacks in children. The administration of atomized inhalation can be selected on an individual basis: salbutamol or terbutaline (weight $\leq 20 \mathrm{~kg}$, $2.5 \mathrm{mg}$ /time; weight $>20 \mathrm{~kg}, 5 \mathrm{mg} /$ time), once every 20 minutes in the first hour. The interval of administration can then be extended according to treatment response every 1-4 hours. A pressurized metered-dose inhaler (pMDI) can also be used for inhalation through a mist storage tank, with single-dose 3-6 sprays/time (<6 years old) and 4-10 sprays/ time ( $\geq 6$ years old), and the interval of administration are the same as for atomized inhalation; children aged $\geq 6$ years with asthma can use ICS combined with long-acting $\beta$ 2 -receptor agonist (LABA) (such as formoterol) as a relief drug.

ICS

During acute mild-to-moderate asthma attacks, ICS (such as budesonide $1 \mathrm{mg} /$ time, twice a day) are used as the initial treatment for their short-acting efficacy on the $\beta 2$ receptor and rapid remission of the attack. Repeated administration every 4-6 hours can be performed as needed, and the administration interval can be gradually extended based on the patient's response to treatment, maintaining for 7-10 days (10).

\section{Management of asthma in the remission period}

To reduce the risk of SARS-CoV-2 infection caused by frequent visits to the hospital, patients should minimize the frequency of outpatient visits and drug collection in severe epidemic areas or at peak times. Asthma control based on symptoms should be emphasized, and family management 
of childhood asthma should be based on the regular evaluation of asthma control and dosage of medications.

\section{Medication in the remission period \\ ICS and leukotriene receptor antagonists (LTRA)}

The original dose is recommended.

\section{Allergen-specific immunotherapy (AIT)}

AIT is given by either sublingual administration (SLIT) or subcutaneous injection (SCIT). During the COVID-19 epidemic, SLIT should be continued at home under the guidance of a physician. During the off-peak period of the epidemic, children with asthma can be treated with SCIT in the hospital as normal. In the most affected areas or during the epidemic peak outbreak, it is suggested to discontinue the treatment in the first 4 weeks of the initial dose stage of SCIT to reduce the risk of acquiring infection from the hospital and during post-injection observation. Four weeks later, SCIT can be delayed for 2 weeks, and the dose can be adjusted according to the specific situation. In the maintenance dose stage, the injection can be postponed for 2 weeks and then be continued according to the original dose. If the delay lasts for more than 2 weeks, the dosage should be readjusted according to the specific circumstances. In principle, postponing for more than 8 weeks is not recommended, and any patients with treatment delays for over 8 weeks should be reinjected from the initial stage.

\section{Biological agents}

Omazumab is currently approved for asthma in children aged $\geq 6$ years in China. In the off-peak phase of the epidemic, the injection can be normally carried out in the hospital at the specified time; in the worse affected areas or at the epidemic peak outbreak, it is recommended to postpone omazumab treatment, and maintain or increase the dosage of the original control medication accordingly.

\section{Self-monitoring and evaluation}

During standard treatment, it is recommended to evaluate the level of asthma control regularly and adjust the treatment plan as necessary. Children with asthma are encouraged to use a peak velocity meter both in the morning and evening every day, to regularly measure peak expiratory velocity and keep an asthma diary to assess daily symptoms. Age-specific tools to access asthma control are advised to access asthma control, including the Childhood Asthma Control Test (C-ACT), Asthma Control Questionnaire (ACQ) and Test for Respiratory and
Asthma Control in Kids (TRACK). During the prevalence of COVID-19, home observation should be made to monitor the signs of asthma attacks such as cough and nasal congestion (11).

\section{Pulmonary function management}

In the stage of epidemic prevention and control, it is recommended to postpone lung function examination. If necessary, the examination should be carried out in a specific negative pressure ward or in negative pressure examination area in addition to meeting the routine indications and excluding contraindications. Strict personal protection, cleaning and disinfection of medical instruments, object surfaces and the environment should be done in line with epidemic prevention and control guidance (12).

\section{Management of coexisting diseases}

Co-existing diseases of childhood asthma include rhinitis, sinusitis, obstructive sleep apnea, gastroesophageal reflux, and obesity, which may affect childhood asthma control. In particular, nasal diseases and symptoms are not only risk factors but also precursors of asthma attacks. Therefore, while staying at home during the coronavirus epidemic, nasal symptoms including nasal obstruction (such as open mouth breathing, sleep drooling, etc.), runny nose, sneezing, nasal itching and sleep snoring should be closely monitored. Nasal diseases should be considered and medication should be administered according to the specific symptoms observed: (I) oral/nasal antihistamine can be used for sneezing, nasal itching or runny nose, and then, step-bystep medicine can be used for symptom control; (II) nasal glucocorticoids (NCS) can be used effectively to alleviate various nasal symptoms caused by rhinitis; however, the medication is slow to take effect; (III) LTRAs can be used to control asthma and nasal symptoms at the same time, and nasal obstruction can be improved more obviously; (IV) oral/nasal decongestants can be used if nasal congestion is serious; however, continuous use should not exceed 7 days; (V) nasal washing with normal saline or $2 \%$ hypertonic saline can remove irritants, allergens and inflammatory secretions in the nose and help improve nasal symptoms; (VI) if the child is diagnosed with allergic rhinitis or other nasal diseases, it is recommended to maintain the current treatment if the condition is well controlled. In cases of recurrence or aggravation of the disease, the medication can be adjusted according to symptoms and the drugs outlined above can be used when necessary. 


\section{Psychological stress and intervention in children with asthma during the COVID-19 epidemic}

Psychological stress in asthmatic children and their parents

Asthma has been considered a typical psychosomatic disease. Environment, state of mind, character and other social and psychological factors are important in the pathogenesis of asthma (13). Overall, $5-20 \%$ of acute attacks are related to emotional factors. Emotional stress can stimulate or aggravate both acute and chronic asthma. For susceptible children, any event that causes emotional stress, e.g., anxiety, depression, anger, excitement, etc. can induce an asthma attack.

During the COVID-19 epidemic, children inevitably have negative emotions such as panic related to the disease, anxiety due to change of routine, and loneliness due to lack of communication with peers. The increased psychological pressure may result in asthma attacks.

The emotions of parents and other guardians of asthmatic children may trigger asthma and may affect the severity of asthma attacks. During the COVID-19 epidemic, parents' psychological pressure increases significantly. Due to home isolation and other reasons, the time parents and children spend together was significantly prolonged, and anxiety about the epidemic and raised concerns on inadequate care of children led to further psychological distress. Some parents are unable to go home for a long period of time due to their involvement in epidemic prevention and control, and some others are separated from children due to isolation measures and infection-related hospitalization. The sudden separation of parents from children significantly reduces parents' care and psychological support to children, negatively affect children's mental state and can give rise to acute attacks and poor control.

\section{Interventions against psychological pressure}

First of all, it is necessary to maintain normal and stable living conditions within the family, to work and rest regularly, and to get enough sleep. Parents should adjust the family atmosphere to maintain emotional stability and pay proper attention to the emotional responses of the child. Parents should try not to be over tense and over concerned, allowing the child to express and vent, to achieve empathetic and understanding, and ultimately learn to manage their emotions. Indoor sports or exercise help to prevent negative emotions and enhance bonding between parents and children. Games, manual work, reading, painting, and housework can improve parent-child relationships and overcome boredom. Parents need to answer children's questions about the epidemic in simple words; coronavirus cartoons and comics help to reduce children's fear and anxiety caused by the unknown and uncertainty, and help children be more confident with hope. Parents unable to accompany their children for a long time should strengthen parent-child communication through telephone, SMS, and voice/video call. Parents can seek guidance and help on various service platforms for psychological consultation, epidemic prevention and control. For children who have serious emotional disturbances or mental abnormalities, it is necessary to consult a professional psychologist (14).

\section{Exercise recommendations for children with asthma during the COVID-19 epidemic}

\section{Childhood asthma and exercises}

Sedentary behavior and reduced physical activity are risk factors for asthma attacks. Increased sedentary behavior may induce decreased forced exhalation volume (FEV) and forced vital capacity (FVC) in children with asthma. Some children are reluctant to take part in physical activity due to fears of an attack, especially those with severe asthma (15). During the COVID-19 epidemic, children are not allowed out for a long time, and they do less exercises than usual, and the risk of acute asthma attacks significantly increases.

\section{Sports recommendation}

Many studies have recommended moderate-to-low intensity aerobic exercise as the primary form of exercise rehabilitation in children with non-acute asthma. Children and parents should not restrict exercise for fear of asthma attacks or difficulty in seeking medical attention.

During the epidemic period, children with asthma should increase physical exercise appropriately, and exercise plans should be made according to age, physical fitness, current health status and hobbies. For less prevalent areas, indoor and outdoor exercise can be combined.

In pandemic areas, communities or buildings are under strict closed management, so outdoor sports cannot be carried out. Various forms of indoor sports can be carried out according to family conditions and children's physical fitness. Importantly, the intensity of physical exercise should not be too high, and the heart rate should be built up 
gradually. Duration of each exercise should be no less than 30 minutes, with 5-10 minutes of warm-up preparation and 5-10 minutes of stretching after exercise. Exercise frequency should not be too high, e.g., 3-4 times a week. The body consumes $20 \%$ more energy in the afternoon and evening than in the morning when taking the same exercise; therefore, timing should be taken into consideration. Exercise should be avoided during an asthma attack or a respiratory infection. If there is a history of exerciseinduced asthma, the specific activity and exercise load should be carefully selected, besides, bronchodilators and LTRA should be used before exercise if necessary (16).

In conclusion, during the COVID-19 epidemic, it is important to provide children with asthma and their parents with the correct guidance. The risk of viral infection can be reduced by wearing masks and washing hands. In addition, childhood asthma can be completely controlled under a series of measures such as reducing exposure to indoor environmental factors, rational medication, active adjustment of psychological states for both children and parents, and moderate exercise.

\section{Acknowledgments}

China Association for Promotion of Health Science and Technology Child Allergy Professional CommitteePenner: Li Sha, Xin Sun, Qinglong Gu, Chuangli Hao; Experts: Children's Hospital Affiliated to Capital Institute Of Pediatrics (Li Sha, Qinglong Gu, Yuzhi Chen, Chuanhe Liu), Xijing Hospital Affiliated to Air Force Medical University (Xin Sun), Children's Hospital of Soochow University (Chuangli Hao, Jianmei Tian, Jinyun Sheng, Yuqing Wang), Peking Union Medical College Hospital, Chinese Academy of Medical Sciences (Kai Guan), Children's Hospital Affiliated to Zhejiang University Medical College (Yong Fu, Lanfang Tang), Children's Hospital Affiliated to Shanghai Jiao Tong University (Xiaoyan Li), People's Hospital of Peking University (Min Wang), Children's Hospital of Chongqing Medical University (Zhou Fu, Zhengxiu Luo), Wuhan Children'S Hospital Affiliated to Tongji Medical College of Huazhong University of Science and Technology (Xiaoxia Lu), Children's Hospital of Kunming Medical University (Hongmin Fu), Shanxi Children's Hospital (Zhiying Han), Chengdu Women's and Children's Central Hospital (Tao Ai), Shandong Provincial Hospital (Xing Chen), The First Affiliated Hospital of Guangzhou Medical University (Dehui Chen). Thanks to Yuting He and Wenjing Gu for organizing and editing this article.

Funding: This work was supported by National Natural Science Foundation of China (No. 81870025) and Suzhou "clinical medical expert team" introduction project (No. SZYJTD201806).

\section{Footnote}

Conflicts of Interest: All authors have completed the ICMJE uniform disclosure form (available at http://dx.doi. org/10.21037/jtd-20-1688). The authors have no conflicts of interest to declare.

Ethical Statement: The authors are accountable for all aspects of the work in ensuring that questions related to the accuracy or integrity of any part of the work are appropriately investigated and resolved.

Open Access Statement: This is an Open Access article distributed in accordance with the Creative Commons Attribution-NonCommercial-NoDerivs 4.0 International License (CC BY-NC-ND 4.0), which permits the noncommercial replication and distribution of the article with the strict proviso that no changes or edits are made and the original work is properly cited (including links to both the formal publication through the relevant DOI and the license). See: https://creativecommons.org/licenses/by-nc-nd/4.0/.

\section{References}

1. National Health Commission of the People's Republic of China. Notice of the national health commission on the revision of the English naming of COVID-19. (2020-0222). Available online: http://www.nhc.gov.cn/xcs/zhengcwj /202002/6ed7614bc35244cab117d5a03c2b4861.shtml

2. National Health Commission of the People's Republic of China. Notice of the general office of the national health commission on the issuance of COVID - 19 prevention and control plan (sixth edition). (2020-03-07). Available online: http://www.nhc.gov.cn/xcs/zhengcwj/202003/4856 d5b0458141fa9f376853224d41d7.shtml

3. National Health Commission of the People's Republic of China. Update on covid-19 outbreak as of 24:00 on 12 April. (2020-04-14). Available online: http://www.nhc. gov.cn/xcs/yqtb/202004/ee6750d722a54876872056e6aa fd5822.shtml

4. Lewis TC, Metitiri EE, Mentz GB, et al. Community Action Against Asthma Steering, Influence of viral 
infection on the relationships between airway cytokines and lung function in asthmatic children. Respir Res 2018;19:228.

5. Raissy HH, Kelly HW. Inhaled corticosteroids in lung disease. Am J Respir Crit Care Med 2013;187:798-803.

6. National Health Commission of the People's Republic of China. Novel coronavirus protection guidelines for infection of people at different risk. (2020-01-31). Available online: http://www.nhc.gov.cn/jkj/s7916/202001/ a3a261dabfcf4c3fa365d4eb07ddab34.shtml

7. Kanchongkittiphon W, Mendell MJ, Gaffin JM, et al. Indoor environmental exposures and exacerbation of asthma: An update to the 2000 review by the Institute of Medicine. Environ Health Perspect 2015;123:6-20.

8. National Asthma Education and Prevention Program. Expert Panel Report 3 (EPR-3): Guidelines for the Diagnosis and Management of Asthma-Summary Report 2007. J Allergy Clin Immunol 2007;120:S94-138.

9. Shim JS, Lee HS, Park DE, et al. Aggravation of asthmatic inflammation by chlorine exposure via innate lymphoid cells and CD11cintermediate macrophages. Allergy 2020;75:381-91.

10. Shen KL, Deng L, Li YZ, et al. Expert consensus on

Cite this article as: China Association for Promotion of Health Science and Technology Child Allergy Professional Committee. Expert recommendations on the management of childhood bronchial asthma during the novel coronavirus pneumonia epidemic. J Thorac Dis 2020;12(8):4391-4397. doi: $10.21037 /$ jtd-20-1688 the use of glucocorticoid aerosol inhalation therapy in pediatrics (revised 2018). J Clin Pediatr 2018;36:95-107.

11. Respiratory group of pediatrics society of Chinese medical association, Editorial board of the Chinese journal of pediatrics. Guidelines for the diagnosis and treatment of bronchial asthma in children (2016 edition). Chin J Pediatr 2016;54:167-81.

12. Gao Y, Ye D, Zheng JP. Guidelines on pulmonary function examination for novel coronavirus (2019-ncov) pneumonia outbreak prevention and control. (2020-01-29). Available online: http://www.sklrd.cn/show.php?id=1351

13. Tully C, Rose M, Breen S, et al. Relationship between parent mood and resilience and child health outcomes in pediatric asthma. Fam Syst Health 2019;37:167-72.

14. Zhang W, Li WM, He ZY, et al. Public psychological protection manual of novel coronavirus. Chendu: Sichuan Science and Technology Press, 2020.

15. Lu KD, Forno E. Exercise and lifestyle changes in pediatric asthma. Curr Opin Pulm Med 2020;26:103-11.

16. Pedersen BK, Saltin B. Exercise as medicine-evidence for prescribing exercise as therapy in 26 different chronic diseases. Scand J Med Sci Sports 2015;25:1-72. 